\title{
Stimulated Emission Depletion Dynamics in Push-Push Polyenes
}

\author{
R. J. Marsh, N. D. Leonczek, D. A. Armoogum, L. Porres", O. Mongin*, M. Blanchard- \\ Desce" $^{*}$ and A. J. Bain ${ }^{+}$
}

\author{
Department of Physics \& Astronomy, University College London, Gower Street, London WC1E 6BT, \\ UK \\ *Équipe Photonique Moléculaire, Institut de Chimie, UMR 6510 SESO, Université de Rennes 1, Campus \\ de Beaulieu, Bât. 10A, 35042 Rennes, Cedex France
}

\begin{abstract}
Two-photon fluorescence polarisation and stimulated emission depletion dynamics are investigated in three high two -photon cross-section push-push polyenes: OM62, LP79 and OM77 and compared to the behaviour of a standard fluorophore (rhodamine 6G). Two-photon fluorescence anisotropy measurements $(\mathrm{R}(0)$ and $\Omega$ ) were undertaken using picosecond time-correlated single photon counting (TCSPC). For OM62 and LP79 these are consistent with a diagonal two-dimensional transition tensor with $\mathrm{S}_{\mathrm{XX}}>\mathrm{S}_{\mathrm{YY}}$. For OM77 the contribution of off-diagonal elements $\left(\mathrm{S}_{\mathrm{XY}}\right.$ $\left.\& S_{Y X}\right)$ appears significant. Two-photon fluorescence anisotropy decay data is combined with streak camera measurements of excited state population depletion to determine stimulated emission cross sections and ground state vibrational relaxation times. Cross-sections for STED in all three polyenes were found to be significantly higher than those for rhodamine 6G. The efficiency of STED is however dependent on the degree to which the $S_{1} \rightarrow S_{0}$ transition is saturated by the DUMP pulse; this is mediated by fast ground state vibrational relaxation. Of the three polyenes, LP79 is seen to combine a large stimulated emission cross-section (c.a. $13 \sigma(\mathrm{r} 6 \mathrm{G})$ ) with rapid ground state relaxation $\left(\tau_{\mathrm{R}}=240 \mathrm{fs}\right)$.
\end{abstract}

Key words: Two-photon absorption, stimulated emission depletion, fluorescence anisotropy, polarisation, vibrational relaxation

\section{INTRODUCTION}

Fluorescence lifetime and anisotropy measurements of molecular probes have found wide application in the studies of biomolecular dynamics and structure ${ }^{1}$. They also show considerable promise as sensitive tools in imaging the dynamics of molecular interactions within cells ${ }^{2,3}$. In this context the application of two-photon excitation to provide both increased spatial and orientational resolution ${ }^{4}$ has proved a notable step change. Stimulated emission depletion of excited states has proved to be a valuable tool in high-resolution molecular spectroscopy ${ }^{5}$ and in the study of ultrafast relaxation in ground and excited electronic states ${ }^{6-9}$. There has been considerable interest in the use of single photon STED in fluorescence microscopy to obtain sub-wavelength image resolution ${ }^{10}$. Recent work in our laboratory has demonstrated the feasibility of performing STED in two-photon excited states ${ }^{11,12}$. We have shown that time-resolved STED measurements circumvent the constraints imposed by the selection rules for single photon emission in that higher degrees of excited state order and correlation functions for molecular motion can be determined ${ }^{13,14}$. Parallel to these developments there has been much recent interest in the development of new fluorescent chromophores optimised for two-photon absorption ${ }^{15}$. Simple quantum models of two-photon absorption in polyenes ${ }^{16-19}$ predict that an elongated conjugated system combined with strong electro-active end groups (electron donating or withdrawing) will lead to a significant enhancement of the two-photon cross-section. In this respect two-photon quadrupolar systems have proved most successful. The design utilises a centro-symmetric system of donor and acceptor groups connected by a conjugated $\pi$-electron system. Compounds based on fluorescent cores such as biphenyl and fluorene have been synthesised and their cross-sections measured ${ }^{20-23}$.

\footnotetext{
${ }^{+}$Corresponding Author, email: a.bain@ucl.ac.uk
} 
In this paper we report preliminary investigations into the two-photon photophysics and stimulated emission depletion dynamics of two fluorene based donor-acceptor-acceptor-donor molecules LP79 ${ }^{24}$ and OM62 ${ }^{25}$ and a branched fluorophore OM77 ${ }^{26}$. The structures of the three fluorophores are shown in figure 1 and their photophysical parameters are listed together with those of rhodamine $6 \mathrm{G}$ in table 1 .

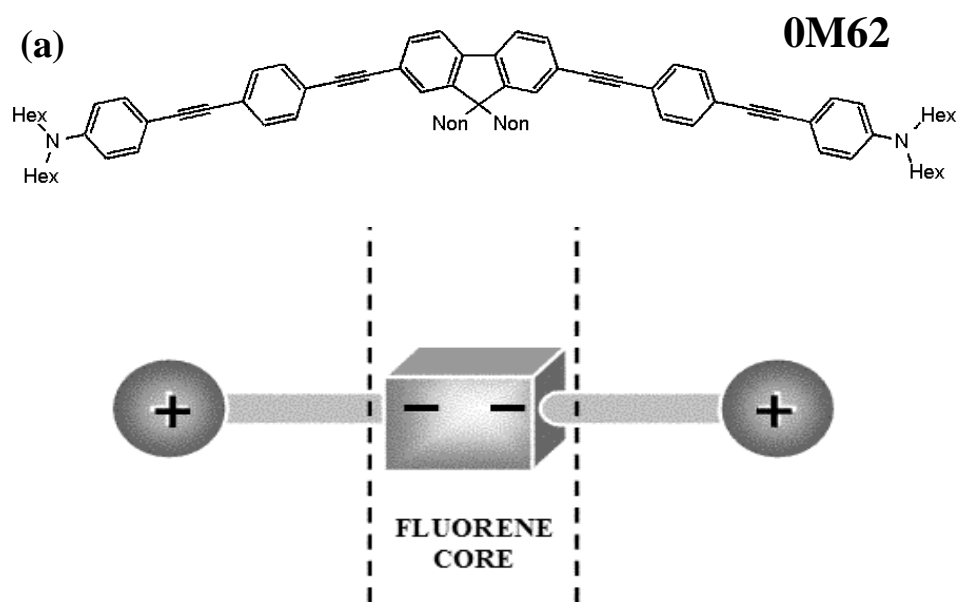

(b)

LP79
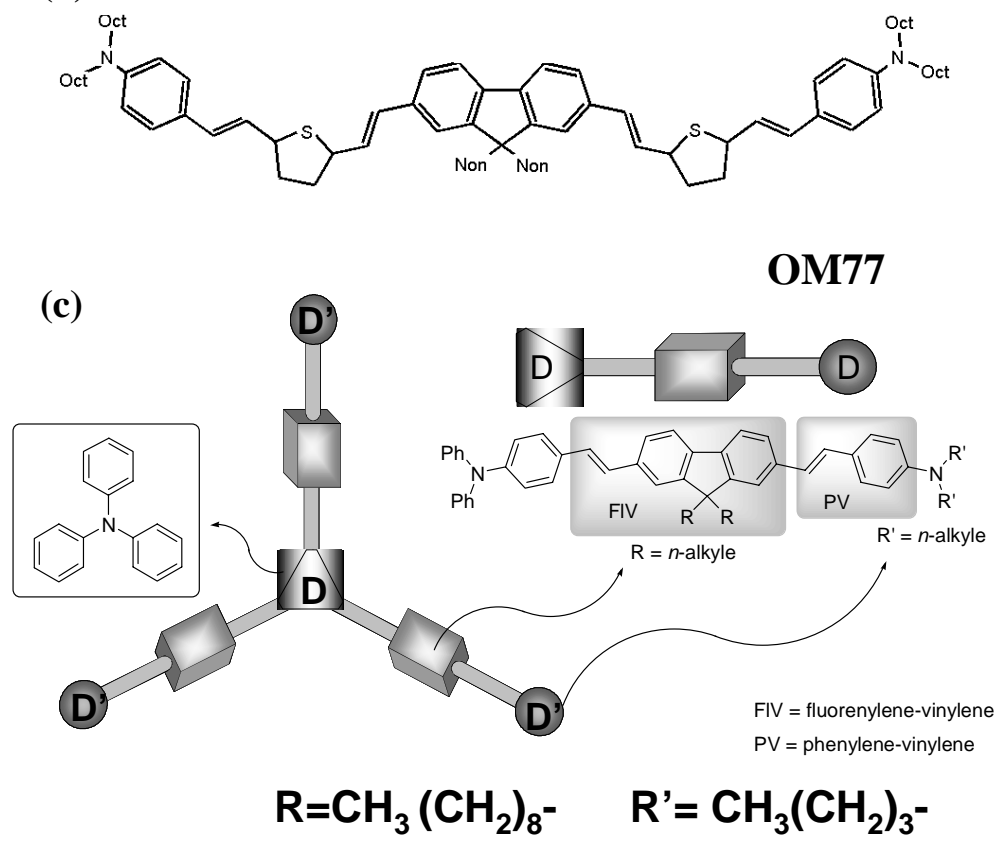

Figure 1: Structures of the two-photon polyenes used in this study (a) OM62 and (b) LP79 are engineered to undergo quadrupolar intramolecular charge redistribution upon two-photon excitation and are based around a fluorene core with electron donating end groups . (c) OM77 consists of a branched (planar) arrangement of three fluorene based quadrupolar chromophores. 


\begin{tabular}{|c|c|c|c|c|}
\hline Fluorophore & OM62 & LP79 & OM77 & Rhodamine 6G \\
\hline Molecular Weight & 1185.876 & 1318.208 & 2267.596 & 479.02 \\
\hline $\begin{array}{c}\text { Single Photon Absorption } \\
\text { Maximum }\end{array}$ & $387 \mathrm{~nm}$ & $470 \mathrm{~nm}$ & $428 \mathrm{~nm}$ & 528 \\
\hline $\boldsymbol{\varepsilon}\left(\mathbf{1 0}^{4} \mathbf{M}^{-1} \cdot \mathbf{c m}^{-1}\right)$ & 12.8 & 12.3 & 24.55 & 11.6 \\
\hline Emission Maxima (nm) & 433.56 & 524.6 & 471.3 & 555 \\
\hline Quantum Yield & 0.82 & 0.47 & 0.77 & 0.95 \\
\hline Fluorescence Lifetime (ns) & 0.692 (in toluene) & 0.869 (in toluene) & 1.12 (in toluene) & $5.7($ ethylene glycol) \\
\hline $\begin{array}{c}\text { TPA Maximum (nm) } \\
\mathbf{\sigma}^{(\mathbf{2})} \text { at TPA Maximum } \\
\left(\mathbf{c m}^{\mathbf{4}} \mathbf{s} / \text { photon) }\right.\end{array}$ & 705 & 705 & 740 & 700 \\
\hline $\begin{array}{c}\mathbf{\sigma}^{(2)} \text { at 800nm } \\
\left(\mathbf{c m}^{4} \mathbf{s} / \text { photon) }\right.\end{array}$ & $4.39 \times 10^{-48}$ & $17.73 \times 10^{-48}$ & $19.82 \times 10^{-48}$ & $0.36 \times 10^{-48}$ \\
\hline
\end{tabular}

Table 1: Photophysical parameters for the three push-push polyenes (LP79, OM62 and OM77) compared to those of rhodamine $6 G$.

\section{POLARISED STIMULATED EMISSION DEPLETION}

A schematic representation of the two-photon STED process is shown in Figure 2. Initial excitation from low lying vibrational levels in the $S_{0}$ ground state occurs via the simultaneous absorption of two (non-resonant) near infra red photons with fast radiationless conversion yielding a vibrationally excited population in the $\mathrm{S}_{1}$ excited electronic state. Fast collisional (and solvent) relaxation leads to the rapid population of lower vibrational levels in $S_{1}$. In the absence of external perturbations the population in $S_{1}$ decays by spontaneous emission to upper vibrational levels of $\mathrm{S}_{0}$ followed by rapid collisional deactivation. In STED a visible laser pulse resonant with the $\mathrm{S}_{1} \rightarrow \mathrm{S}_{0}$ emission is applied to induce transitions to upper vibrational levels of $\mathrm{S}_{0}$. The net result of this process is a sharp reduction in the excited state population (fluorescence intensity) and a change in molecular alignment (fluorescence anisotropy) dependent on the relative polarisation of the two laser pulses. Depletion of the excited state is maximised for parallel PUMP and DUMP polarisations and at PUMP-DUMP delays that are short in comparison to the rotational diffusion time of the molecule ${ }^{11,12}$. STED dynamics can be modelled analytically in the limit of fast ground state relaxation and weak depletion of the excited state population. For strong saturation of the DUMP transition, analytic solutions to the coupled rate equations are possible for model DUMP pulse shapes ${ }^{11,12}$.

The rate equations for STED on a timescale that is fast compared to spontaneous emission (nanosecond to sub-nanosecond) and orientational relaxation (nanosecond) are given by

$$
\begin{aligned}
& \frac{d N_{E X}(\theta, t)}{d t}=-\frac{I_{D}(t) \sigma W_{D}(\theta)}{h v}\left(N_{E X}(\theta, t)-N_{G S}(\theta, t)\right) \\
& \frac{d N_{G S}(\theta, t)}{d t}=\frac{I_{D}(t) \sigma W_{D}(\theta)}{h v}\left(N_{E X}(\theta, t)-N_{G S}(\theta, t)\right)-\frac{N_{G S}(\theta, t)}{\tau_{R}}
\end{aligned}
$$


Where $N_{E X}(\theta, t)$ and $N_{G S}(\theta, t)$ are the ground and excited state populations, $I_{D}(t)$ is the DUMP pulse intensity, $\mathrm{W}_{\mathrm{D}}(\theta)$ is the angular dependence of the DUMP probability, $\sigma$ is the stimulated emission cross-section $\left(\mathrm{cm}^{2}\right)$ and $\tau_{\mathrm{R}}$ is the ground state vibrational relaxation time. It is possible to obtain analytic solutions to the rate equations by assuming a rectangular DUMP pulse with width $\tau_{\mathrm{P}}$, which yield the fractional remaining population $\mathrm{F}_{\mathrm{R}}$ as a function of $\mathrm{E}_{\mathrm{P}} \sigma / \mathrm{h} v \mathrm{~A}$ for given ratios of $\tau_{P} / \tau_{R}$. Given that the initial population distribution in the upper ground state vibrational levels is negligible, the excited state population evaluated at $\mathrm{t}=\tau_{\mathrm{P}}$ is,

$$
N_{E X}\left(\theta, \tau_{P}\right)=\frac{N_{E X}(\theta, 0)}{2 d}\left[\exp \left(-\frac{1}{2}\left\{\frac{\tau_{P}}{\tau_{R}}+2 S \cos ^{2} \theta+d\right\}\right)\right]\left\{\frac{\tau_{P}}{\tau_{R}}\left(e^{d}-1\right)+d\left(e^{d}+1\right)\right\}
$$

where the parameter $\mathrm{d}$ is given by

$$
d=\sqrt{\left(\tau_{P} / \tau_{R}\right)^{2}+4 S^{2} \cos ^{4} \theta}
$$

The fractional depletion of the excited state population can be calculated from

$$
1-F_{R}=\int_{0}^{2 \pi} \int_{0}^{\pi}\left[N_{E X}\left(\theta, \tau_{P}\right)-N_{E X}(\theta, 0)\right] \sin \theta d \theta d \varphi / \int_{0}^{2 \pi} \int_{0}^{\pi} N_{E X}(\theta, 0) \sin \theta d \theta d \varphi
$$

where $N_{E X}(\theta, 0)$ is the excited state population immediately prior to STED. The evaluation of equation 5 was performed using a Mathematica programme. This yields numerical solutions for the population removal immediately following STED (i.e. before the onset of significant orientational relaxation) for varying $\mathrm{S}$ and ratios of DUMP pulse-width to ground state vibrational relaxation time $\left(\tau_{\mathrm{P}} / \tau_{\mathrm{R}}\right)$.
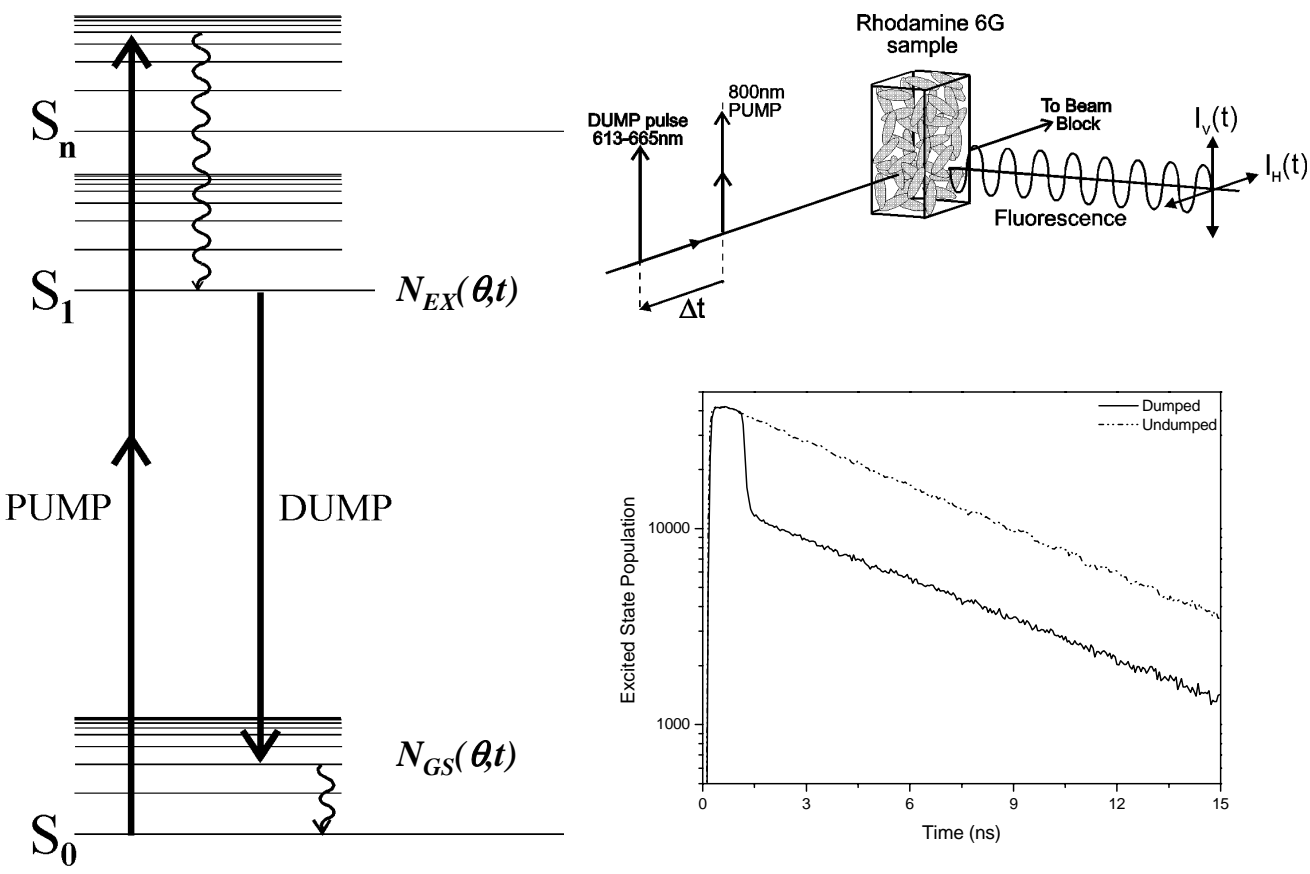

Figure 2: Stimulated emission depletion (STED) in rhodamine 6G. Linearly polarised two-photon excitation (PUMP) is followed by a time delayed (1ns, 17nJ, 622nm) DUMP pulse. With parallel PUMP and DUMP polarisations a $70 \%$ reduction in the excited state population $(\Delta N / N)$ is achieved. 
A set of numerical solutions for $F_{R}$ with $S$ for different values of $\tau_{\mathrm{P}} / \tau_{R}$ is shown in figure 3. This shows that as the DUMP pulse length is increased (i.e. $\tau_{\mathrm{P}} / \tau_{\mathrm{R}}$ rises) re-pumping of $S_{1}$ is reduced and higher levels of excited state depletion can be achieved. By fitting numerical solutions as in figure 3 to experimental data, it is possible to obtain values for the stimulated emission cross-section and ground state vibrational relaxation times ${ }^{11,12}$.

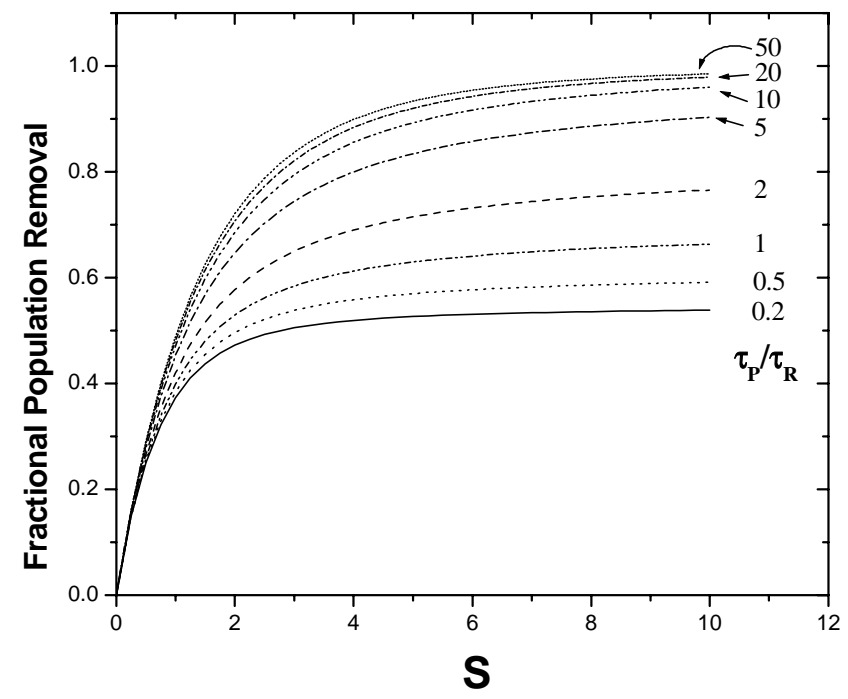

Figure 3: Numerical solutions to the rate equations generate a family of saturation curves for population removal by $S T E D$ for different values of $\tau_{P} / \tau_{R}$. In this example, $\tau_{P} / \tau_{R}$ is varied from 0.2 to 50 and values of $S$ from 0 to 12.

\section{POLARISED TWO-PHOTON EXCITATION}

The transition probability for the absorption of two photons of polarisations $\alpha$ and $\beta$ between states $i$ and $f$ is proportional to the squared modulus of the tensor $\boldsymbol{W}_{\alpha \beta}^{i \rightarrow f}$ defined by a sum of transitions involving intermediate (virtual) states $\mathrm{n}^{26}$,

$$
\left|\boldsymbol{W}_{\alpha \beta}^{i \rightarrow f}\right|^{2}=\mid \sum_{n}\left(\frac{\alpha \cdot\langle\boldsymbol{i}|\boldsymbol{r}| \boldsymbol{n}\rangle\langle\boldsymbol{n}|\boldsymbol{r}| \boldsymbol{f}\rangle \cdot \beta}{v_{n}-v_{\alpha}+i \Gamma_{n}}+\left.\frac{\beta \cdot\langle\boldsymbol{i}|\boldsymbol{r}| \boldsymbol{n}\rangle\langle\boldsymbol{n}|\boldsymbol{r}| \boldsymbol{f}\rangle \cdot \alpha}{v_{n}-v_{\beta}+i \Gamma_{n}}\right|^{2}\right.
$$

where $v_{\mathrm{n}}$ and $\Gamma_{\mathrm{n}}$ are the transition frequency and homogeneous linewidth of $\mathrm{n}$ respectively. In the molecular frame of reference the transition tensor can have 9 elements $\mathrm{S}_{\mathrm{AB}}$ where $\mathrm{A}, \mathrm{B}=\mathrm{X}, \mathrm{Y}$ or $\mathrm{Z}$,

$$
\left|W_{\alpha \beta}^{i \rightarrow f}\right|^{2} \equiv\left|\sum_{A, B=X, Y, Z} S_{A B}\right|^{2}
$$

For degenerate two-photon absorption $\mathrm{S}_{\mathrm{AB}}=\mathrm{S}_{\mathrm{BA}}$ For planar aromatic molecules the two-photon transition is dominated by moments in the plane of the molecule ${ }^{27}$. Unlike single photon transitions from an isotropic ground state the two-photon absorption probability is dependent on the relative polarisations of the two photons. The relative absorption strengths for linear and circular polarised two-photon absorption define the parameter $\Omega$

$$
\Omega=I_{A B S}^{C} / I_{A B S}^{L}=\int I_{F}^{C}(t) d t / \int I_{F}^{L}(t) d t
$$

This can be determined by total fluorescence intensity measurements as shown in figure 4 . 


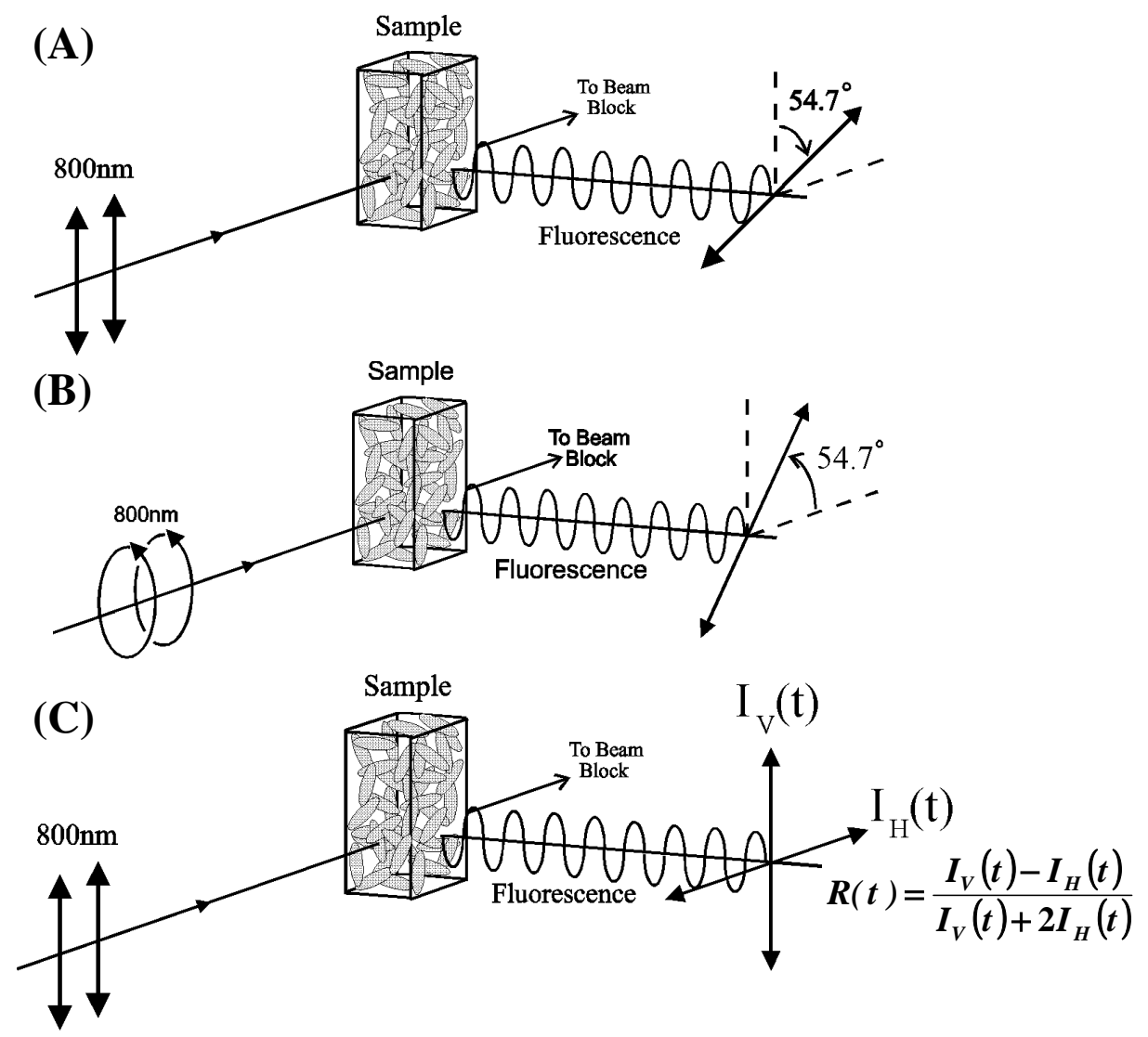

Figure 4: Excitation-detection geometries for the determination of the transition strength for (A) linearly polarised and (B) circularly polarised two-photon excitation. Fluorescence intensity measurements are made at magic angle polarisation settings with respect to the quantisation (symmetry) axis for the two polarisations. Alternatively, the fluorescence intensity can be constructed from $I_{V}(t)+2 I_{H}(t)$ and $2 I_{V}(t)+I_{H}(t)$ measurements for linear and circular polarisations respectively. $(C)$ : Excitation-detection geometry for fluorescence anisotropy measurements following linearly polarised two-photon excitation

In terms of a planar transition tensor where the emission transition dipole moment lies along the molecular axis, $\Omega$ is given by ${ }^{26}$

$$
\Omega=\frac{1}{2} \frac{\left(S_{X X}+S_{Y Y}\right)^{2}+3\left(S_{X X}-S_{Y Y}\right)^{2}+12 S_{X Y}^{2}}{2\left(S_{X X}+S_{Y Y}\right)^{2}+\left(S_{X X}-S_{Y Y}\right)^{2}+3 S_{X Y}^{2}}
$$

The fluorescence anisotropy is also dependent on the structure of the transition tensor, for linearly polarised excitation the initial fluorescence anisotropy as measured in figure 4 is given by ${ }^{26}$,

$$
R(0)=\frac{I_{V}(0)-I_{H}(0)}{I_{V}(0)+2 I_{H}(0)}=\left(\frac{1}{7}\right) \frac{2\left(S_{X X}+S_{Y Y}\right)^{2}+\left(S_{X X}-S_{Y Y}\right)^{2}+4 S_{X Y}^{2}+9\left(S_{X X}^{2}-S_{Y Y}^{2}\right)}{2\left(S_{X X}+S_{Y Y}\right)^{2}+\left(S_{X X}-S_{Y Y}\right)^{2}+3 S_{X Y}^{2}}
$$

For a diagonal transition tensor $\mathrm{S}_{\mathrm{XY}}=0, \Omega$ and $\mathrm{R}(0)$ are determined solely by the ratio $\mathrm{S}=\mathrm{S}_{\mathrm{YY}} / \mathrm{S}_{\mathrm{XX}}$, 


$$
\begin{aligned}
& \Omega=\left(\frac{1}{2}\right) \frac{(1+S)^{2}+3(1-S)^{2}}{2(1+S)^{2}+(1-S)^{2}} \\
& R(0)=\left(\frac{1}{7}\right) \frac{2(1+S)^{2}+(1-S)^{2}+9\left(1-S^{2}\right)}{2(1+S)^{2}+(1-S)^{2}}
\end{aligned}
$$

The variation in $\Omega$ and $\mathrm{R}(0)$ with $\mathrm{S}$ is displayed in figure 5 . Where the transition is largely dominated by $\mathrm{S}_{\mathrm{Xx}}$ an initial fluorescence anisotropy of $4 / 7$ is obtained that is characteristic of a $\cos ^{4} \theta$ distribution of transition dipoles in the molecular frame; the corresponding value for $\Omega$ is $2 / 3$. Deviations from these values are indicative of contributions from $\mathrm{S}_{\mathrm{YY}}$ and/or Sxy.

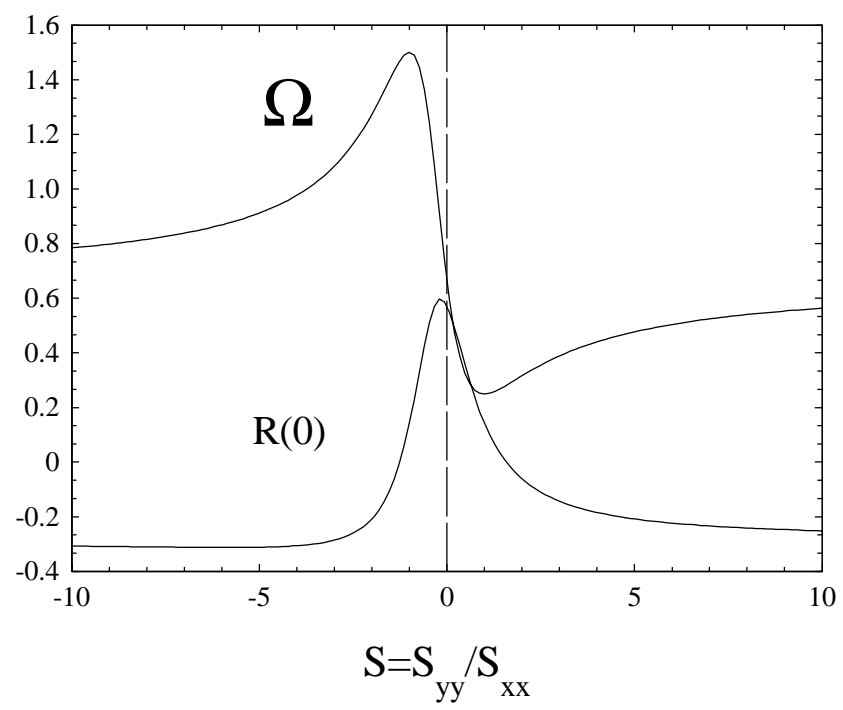

Figure 5: Variation in the absorption ratio $\Omega$ and the initial anisotropy $R(0)$ with the ratio $S=S_{Y \mid Y} / S_{X X}$ for a diagonal planar two-photon transition tensor. The dashed line at $S=0$ intersects the curves at $\Omega=2 / 3$ and $R(0)=4 / 7$.

\section{EXPERIMENTAL PROCEDURE}

The experimental apparatus for STED and two-photon anisotropy measurements is illustrated in fig. 6 and has been described in detail elsewhere ${ }^{11,12}$. The samples were contained in $1 \mathrm{~cm}$ quartz cuvettes (Hellma) with four optical windows. Two-photon excitation (PUMP) was achieved using the partial output of a regeneratively amplified Ti: Sapphire laser (Coherent Mira 900F, Coherent RegA 9000) which provided 800nm laser pulses with up to 60nJ energy and $140 \mathrm{fs}$ FWHM pulse width at a repetition rate of $250 \mathrm{KHz}$. The majority of the $800 \mathrm{~nm}$ output (ca. $3.4 \mu \mathrm{J}$ ) was used to pump an optical parametric amplifier (Coherent OPA 9400) which provided a synchronous (ca. 250fs) pulse train tuneable across the visible spectrum $(450-700 \mathrm{~nm})$. To prevent saturation at low DUMP energies (c.a. $1 \mathrm{~nJ}$ ) and DUMP induced two-photon fluorescence the OPA pulses were stretched using the material dispersion provided by passage through a distilled water cell. With this arrangement OPA pulses from $250 \mathrm{fs}$ to $3 \mathrm{ps}$ could be produced. The PUMP and DUMP wavelengths were monitored using a laser spectrum analyser (IST-Rees) and the pulse widths measured using a scanning autocorrellator (APE). PUMP and DUMP beam polarisations were controlled using broadband half waveplates (Melles-Griot) and a quarter waveplate (Special Optics). The PUMP-DUMP separation could be set and scanned using a variable optical delay line (Time \& Precision). PUMP and DUMP powers were controlled by neutral density wheels and measured by a precision power meter (Anritsu). Prior to the sample the PUMP and DUMP beams were spatially overlapped using a broadband dichroic beam combiner (CVI Optics), passed through an aperture of fixed diameter $(2.2 \mathrm{~mm})$ and focussed into the sample using a $2.54 \mathrm{~cm}$ 
achromatic lens (Melles-Griot). Scattered laser light was blocked using appropriate interference filters (Corion LS600, LS550 \&500) and glass cut-off filters (Schott BG39). A glass cut-off filter (Schott OG590) was placed in the DUMP path before the sample to block short-wavelength remnants of white continuum generated in the OPA. Population depletion measurements employed parallel PUMP and DUMP polarisations and used either a streak camera (Hamamatsu C4334) with a "magic angle" polariser setting, or total intensity measurements constructed from vertical and horizontally polarised decays recorded by the time correlated single photon counting (TCSPC) system

${ }^{28}$. The TCSPC system was also used to record two-photon fluorescence lifetime and anisotropy measurements used in the analysis of the STED data. Fine adjustment of the PUMP and DUMP powers were undertaken using neutral density wheels (Melles Griot). Typical on-sample PUMP energies were less than 30nJ (for LP79 and OM77 excitation pulse energies were c.a. $5 \mathrm{~nJ}$ and $12 \mathrm{~nJ}$ respectively), on-sample DUMP powers were varied between 0 and $33 \mathrm{~nJ}$.

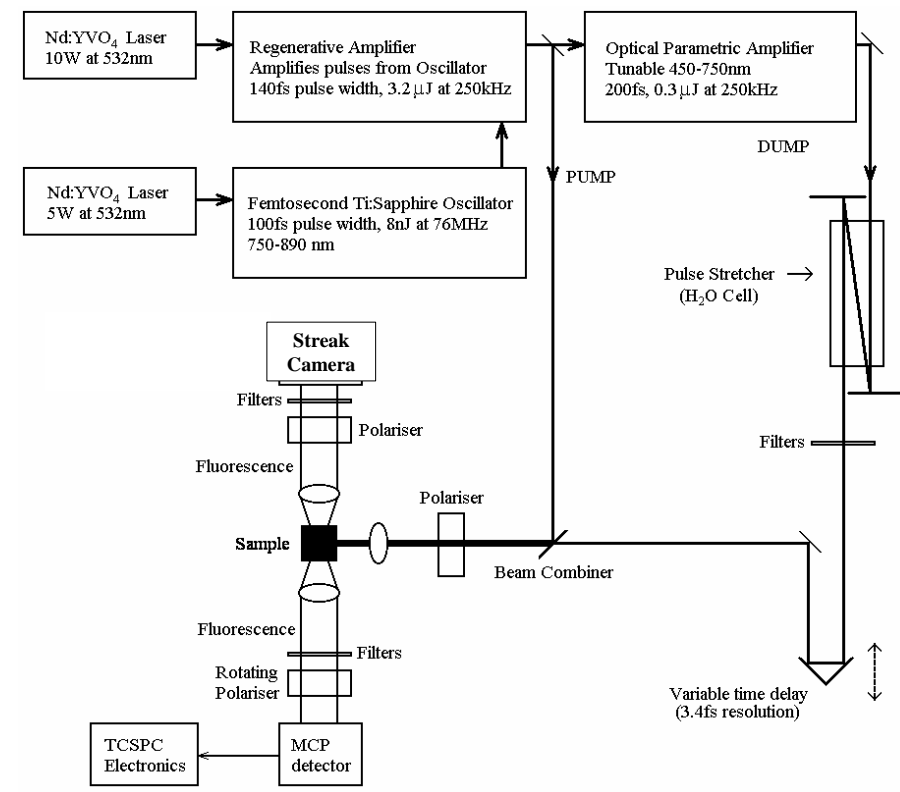

Figure 6: Schematic diagram of the femtosecond laser system and $90^{\circ}$ excitationdetection geometry used in the STED and twophoton anisotropy experiments. Group velocity dispersion from a distilled water cell is used to stretch the DUMP pulses from 200fs to ca. 900fs-2.5ps thus reducing both the saturation of the DUMP transition and the background caused by secondary two-photon excitation.

\section{RESULTS}

\section{TWO-PHOTON ANISOTROPY MEASUREMENTS}

Two-photon absorption strengths were measured for linear and circularly polarised two-photon excitation of rhodamine 6G (in ethylene glycol $5 \times 10^{-4} \mathrm{M}$ ), LP79, OM62 and OM77 (all in toluene c.a. $10^{-4} \mathrm{M}$ ) using time correlated single photon counting with subsequent integration of the fluorescence intensity decays. Two-photon fluorescence anisotropy decays were also recorded using the same experimental arrangement. The $\Omega$ and $\mathrm{R}(0)$ values recorded for the molecules are set out in table 2. From table 2 it is clear that rhodamine $6 \mathrm{G}$ most closely follows the behaviour characterised by a diagonal transition tensor in which $S_{X X}>>S_{Y Y}$. The initial fluorescence anisotropies for OM62 and LP79 are close to those for rhodamine 6G. However, the $\Omega$ values are both slightly above the theoretical ratio of $2 / 3$ for $S=0$. The ratio of the two anisotropies $R(0) / \Omega$ from [11] and [12] is given by

$$
\frac{R(0)}{\Omega}=\left(\frac{2}{7}\right) \frac{2(1+S)^{2}+(1-S)^{2}+9\left(1-S^{2}\right)}{(1+S)^{2}+3(1-S)^{2}}
$$


For a planar diagonal transition tensor this quantity can be used to calculate S. Since [13] has two roots, these can be used via [11] and [12] to calculate values for $\mathrm{R}(0)$ and $\Omega$ which can be compared to the measured values in table 2 .

\begin{tabular}{|c|c|c|c|}
\hline & $\mathbf{R}(\mathbf{0})$ & $\Omega$ & $\tau_{\text {Rot }}($ room temp $)$ \\
\hline $\begin{array}{c}\text { 2-D Planar } \\
\text { Transition } \\
S_{\mathrm{XY}}=\mathbf{S}_{\mathrm{YY}}=\mathbf{0}\end{array}$ & $0.5714(4 / 7)$ & $0.67(2 / 3)$ & \\
\hline Rhodamine 6G & $0.531 \pm 0.008$ & $0.65 \pm 0.04$ & $2.34 \pm 0.05 n s$ (ethylene glycol) \\
\hline OM62 & $0.50 \pm 0.03$ & $0.76 \pm 0.04$ & $1.18 \pm 0.15 n$ ns (toluene) \\
\hline LP79 & $\mathbf{0 . 5 2} \pm \mathbf{0 . 0 3}$ & $0.73 \pm 0.01$ & $1.06 \pm 0.11$ ns (toluene) \\
\hline 0M77 & $0.16 \pm 0.03$ & $0.81 \pm 0.02$ & $0.802 \pm 0.08 n$ (toluene) \\
\hline
\end{tabular}

Table 2: Initial Anisotropy $(R(0))$ and absorption anisotropy ratios $(\Omega)$ obtained for rhodamine $6 G$, OM62, LP79 and OM77 together with those expected for a planar transition dominated by $S_{X X}$. The fluorescence anisotropy decay $R(t)$ was found to follow single-exponential relaxation for all the molecules with relaxation times $\left(\tau_{\text {Rot }}\right)$ c.a. Ins in toluene and c.a. 2 ns in ethylene glycol.

\begin{tabular}{|c|c|c|c|c|c|c|c|}
\hline & $\mathbf{R}(\mathbf{0}) / \Omega$ & $\mathrm{S}_{-}$ & $\Delta \mathbf{R}(\mathbf{0}) \mathrm{S}$. & $\Delta \Omega \mathrm{S}$ & $\mathrm{S}_{+}$ & $\Delta \mathbf{R}(\mathbf{0}) \mathrm{S}_{+}$ & $\Delta \Omega \mathrm{S}_{+}$ \\
\hline Rhodamine 6G & $\mathbf{0 . 8 2} \pm \mathbf{0 . 0 5}$ & $\mathbf{- 0 . 0 4 0}$ & $\mathbf{- 0 . 0 5}$ & $\mathbf{0 . 0 5}$ & $\mathbf{0 . 8 1}$ & $\mathbf{0 . 4 6}$ & $\mathbf{0 . 3 9}$ \\
\hline LP79 & $\mathbf{0 . 7 1} \pm \mathbf{0 . 0 4}$ & $\mathbf{- 0 . 0 1 4}$ & $\mathbf{- 0 . 0 8}$ & $\mathbf{- 0 . 0 9}$ & $\mathbf{0 . 9 0}$ & $\mathbf{0 . 5 5}$ & $\mathbf{0 . 4 8}$ \\
\hline OM62 & $\mathbf{0 . 6 6} \pm \mathbf{0 . 0 5}$ & $\mathbf{- 0 . 1 9 5}$ & $\mathbf{- 0 . 0 9}$ & $\mathbf{- 0 . 1 4}$ & $\mathbf{0 . 9 4}$ & $\mathbf{0 . 6 0}$ & $\mathbf{0 . 5 1}$ \\
\hline OM77 & $\mathbf{0 . 2 0} \pm 0.04$ & $\mathbf{- 0 . 8 0 7}$ & $\mathbf{- 0 . 0 4}$ & $\mathbf{- 0 . 6 6}$ & 1.33 & $\mathbf{0 . 7 6}$ & $\mathbf{0 . 5 5}$ \\
\hline
\end{tabular}

Table 3: Values of $R(0) / \Omega$ obtained for rhodamine $6 G$ and the three two-photon polyenes. These are used to determine the two possible values of $S\left(S . \& S_{+}\right)$, which in turn can be used to calculate values of $R(0)$ and $\Omega$. The deviations of these from experimental measurements are tabulated and used to assess the structure of the transition tensor and the applicability of the $2 D$ diagonal model.

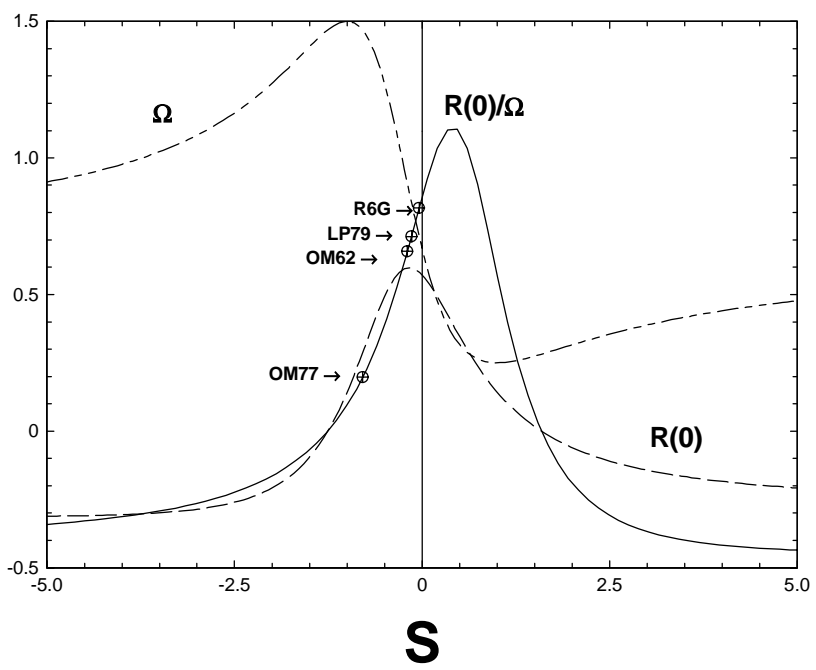

Figure 7: Variation in the anisotropy ratio $R(0) / \Omega$ with $S$ (solid line) together with the $S$-dependence of $R(0)$ and $\Omega$ (dashed lines). Experimental values of $R(0) / \Omega$ and the corresponding $S$. values are superimposed on the theoretical $R(0) / \Omega$ curve. 
From table 3 it is clear that for rhodamine 6G, LP79 and OM77 the polarisation measurements are consistent with a diagonal transition tensor for which $S$ is either close to zero, or of negative sign with $\left|S_{X X}\right|>\left|S_{Y Y}\right|$, these values of $S$ are plotted in figure 7. For OM77 the values of $\mathrm{S}$ obtained do not correspond well with both $\mathrm{R}(0)$ and $\Omega$

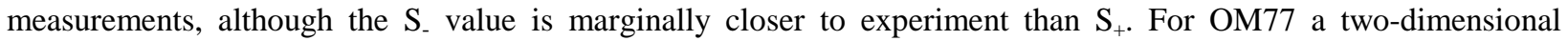
diagonal tensor is not an adequate description of the transition. Given the arrangement of three equivalent chromophores in the X-Y plane off-diagonal elements can be expected and the contribution of Z-polarised tensor components could arise from twisting of the individual chromophores.

In addition to the initial fluorescence anisotropy and $\Omega$ measurements, anisotropy decays were measured for the four fluorophores. All were found to exhibit single exponential dynamics whose relaxation times $\tau_{\text {Rot }}$ are listed in table 4. The lack of any measurable bi-exponential component in LP79 and OM62C is indicative of rotational diffusion of an emission dipole array aligned along the symmetry axis of the molecule. The low initial anisotropy in OM77 may arise from the structure (off-diagonal or non-planar moments) of the transition tensor or from depolarisation mechanisms within the fluorescing $\left(\mathrm{S}_{1}\right)$ state. To this end a comparison between single and twophoton fluorescence anisotropy data would be instructive. In addition to providing useful structural information on the two-photon transition, fluorescence anisotropy measurements are a necessary input into the determination of STED cross-sections and ground state relaxation times. The efficiency of STED is strongly dependent on the orientation of the transition dipole moment and the DUMP polarisation vector ${ }^{11,12}$. For isotropic rotational diffusion (assuming a planar two-dimensional diagonal transition tensor with $\mathrm{S}_{\mathrm{YY}}=0$ ), the orientational distribution at time $\mathrm{t}$ following the application of the PUMP pulse is given by,

$$
\mathrm{N}_{\mathrm{EX}}(\theta, \phi, t)=\mathrm{N}_{\mathrm{EX}} \exp \left(-\frac{t}{t_{f}}\right) \frac{1}{\sqrt{4 \pi}}\left[Y_{00}+\frac{20}{7 \sqrt{5}} Y_{20}(\theta, \phi) \exp \left(-\frac{t}{\tau_{\text {Rot }}}\right)+\frac{8}{21} Y_{40}(\theta, \phi) \exp \left(-\frac{10 t}{3 \tau_{\text {Rot }}}\right)\right]
$$

The determination of $\tau_{\text {Rot }}$ is used via [14] as input to [3] with correction factors to the $Y_{20}$ and $Y_{40}$ terms to account for the deviation of the distribution function from that predicted by theory ${ }^{29}$.

\section{STED DEPLETION DYNAMICS}

Population depletion measurements for the four fluorophores are shown in figure 8 together with fits to the data using equations [3]-[5] and [14]. This approach yields a cross-section $(\sigma)$ for STED $\left(\mathrm{cm}^{2}\right)$ and the ground state vibrational relaxation time $\left(\tau_{R}\right){ }^{11,12}$. The values obtained are displayed in table 3 . Clearly the most efficient fluorophore for STED is LP79 which has a substantial STED cross section that is over an order of magnitude larger than that obtained for the optimal DUMP transition wavelength in rhodamine 6G. Moreover, STED in LP79 is further assisted by rapid relaxation of the lower levels of the DUMP transition (240fs). This is in contrast to OM77, which whilst possessing a large STED cross section has both slow ground state vibrational relaxation (c.a. 900fs) and a low initial anisotropy. In the linear depletion limit, the degree of population depletion is proportional to the degree of excited state alignment ${ }^{11,12}$,

$$
1-F_{R}(t)=(1+2 R(t)) \sigma E / 3 A h v
$$

In this limit the intrinsic STED efficiency can be defined in terms of an effective cross section

$$
\sigma_{E F F}=(1+2 R(0)) \sigma / 3
$$

Values of $\sigma_{\mathrm{EFF}}$ are displayed in table 8. From this data it is clear that linear depletion in LP79 is most efficient. Ground state relaxation is however an important factor. It is instructive to note that $80 \%$ depletion in LP79 is realised by less than 2nJ DUMP energy (at a spot size of $1.77 \times 10^{-6} \mathrm{~cm}^{2}$ ), the onset of saturation (determined by $\tau_{\mathrm{P}} / \tau_{\mathrm{R}}$ ) occurs 
at c.a. 1-1.5nJ; the corresponding PUMP energy is c.a. $5 \mathrm{~nJ}$. In the other fluorophores, equivalent levels of depletion are only achievable at higher DUMP energies that are well outside the linear depletion regime.
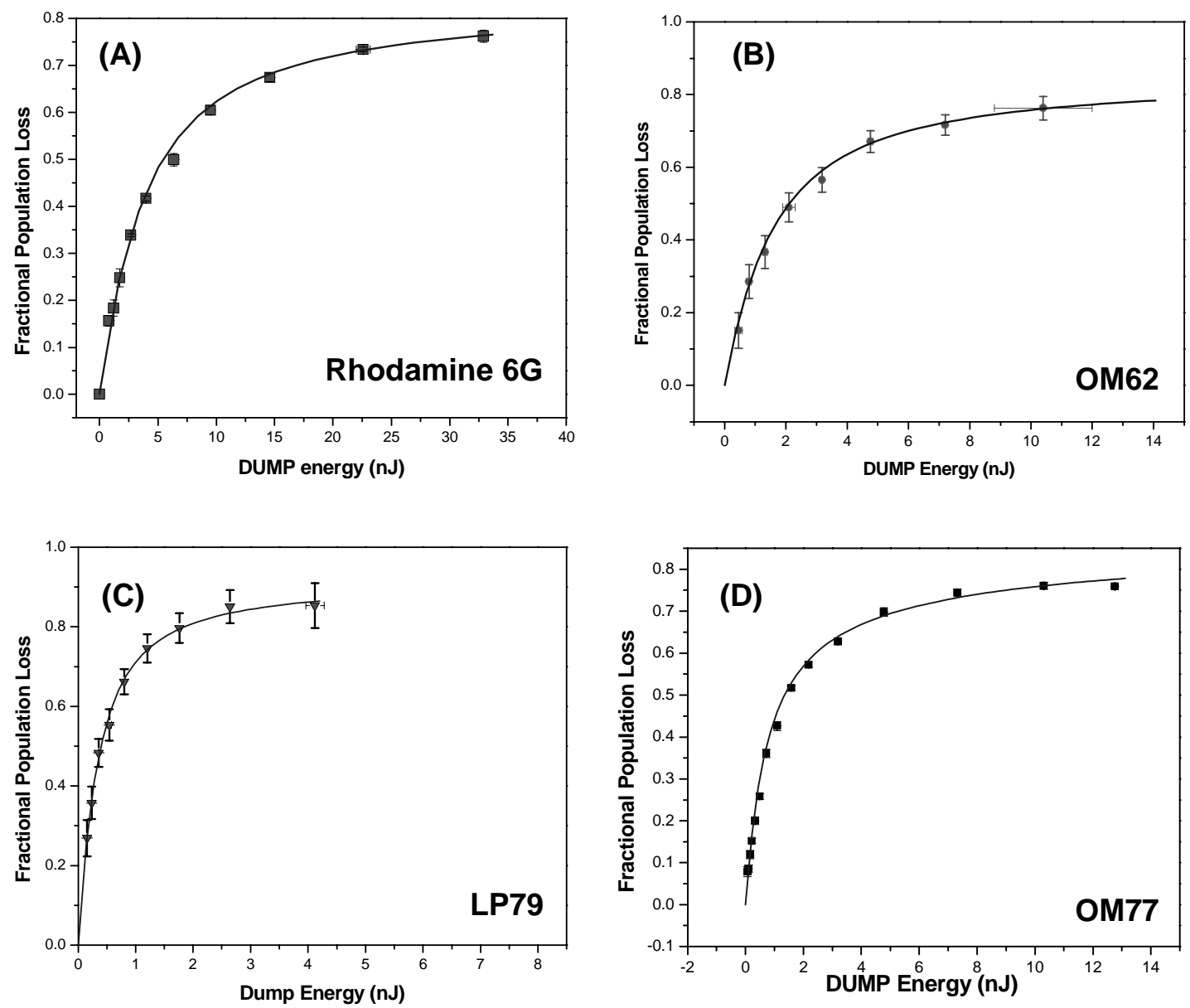

Figure 8: Saturation of STED in (A): Rhodamine 6G (B): OM62, (C): LP79 and (D): OM77. Excited state population depletion becomes less effective with increasing DUMP pulse energy as re-pumping of $S_{1}$ becomes more pronounced due to the finite lifetime of the $S_{0}$ vibrational levels. Solid lines indicate a fit to the data using [12]

\begin{tabular}{|c|c|c|c|}
\hline & $\sigma\left(10^{-16} \mathrm{~cm}^{2}\right)$ & $\tau_{\mathrm{R}}(\mathrm{fs})$ & $\sigma_{\mathrm{EFF}=}(1+2 \mathrm{R}(0)) \sigma / 3\left(10^{-16} \mathrm{~cm}^{2}\right)$ \\
\hline Rhodamine 6G & $1.65(\lambda=634 \mathrm{~nm})$ & 365 & 1.13 \\
\hline OM62 & $4.57(\lambda=546 \mathrm{~nm})$ & 979 & 3.05 \\
\hline OM77 & $14.8(\lambda=545 \mathrm{~nm})$ & 848 & 6.47 \\
\hline LP79 & $21.6(\lambda=580 \mathrm{~nm})$ & $\mathbf{2 4 0}$ & 14.68 \\
\hline
\end{tabular}

Table 4: Stimulated emission cross-sections and ground state vibrational relaxation times obtained from the STED saturation data of figure 8 . Values for the effective STED cross-section $\left(\sigma_{\mathrm{EFF}}\right)$ are calculated from [16] using the initial anisotropy data in Table 2 


\section{CONCLUSIONS}

Two-photon fluorescence polarisation and stimulated emission depletion dynamics have been investigated for three high two-photon cross-section push-push polyenes: OM62, LP79 and OM77 and compared to the behaviour of rhodamine 6G. Two-photon fluorescence anisotropy measurements have yielded information on the structure of the two-photon transition tensor and are used together with population depletion measurements to determine stimulated emission cross sections and ground state vibrational relaxation times. LP79 is seen to combine a large two-photon absorption cross-section with a high $\sigma_{\mathrm{EFF}}$ and a fast ground state vibrational relaxation time. The possibility of achieving efficient two-photon excitation and STED with sub-nanojoule energies is clearly possible in such systems and makes them attractive candidates for unamplified laser applications such as two-photon STED microscopy.

\section{REFERENCES}

[1] N Chadborn J Bryant, A J Bain and P O’Shea, Biophysical J. 76, 2198-2207 1999

[2] V Calleja., S M Ameer-Beg, B Vojnovic., R Woscholski, J Downward and B Larijani, Biochem. J. 372, 33-40 2003

[3] Clayton A. H., Hanley Q. S., Amdt-Jovin A. J., Subramanian V. and Jovin T. M., Biophysical J. 83, 1631-1649 2002

[4] W Denk, J H Strickler and W W Webb, Science 248, 73-76 1990

[5] D E Reisner, R W Field, J L Kinsey and H-L Dai, J. Chem. Phys. 80, 5968-5978 1984

[6] Q H Zhong, Z Wang, Y Sun., Q Zhu, F Kong, Chem. Phys. Lett. 248, 277-282 1996

[7] S. A. Kovalenko, J. Ruthmann and N. P. Ernsting, J. Chem. Phys. 109, 1894-1900 1998

[8] G Haran, E A Morlino, J Matthes,R Callendar and R M Hochstrasser, J. Phys. Chem. A 103, 2202-2207 1999

[9] P Changenet-Barret C T Choma, E F Gooding, W F DeGrado and R M Hochstrasser, J. Phys. Chem. B, 104, $9322-9329$ 2000

[10] T A Klar, S Jakobs, M Byba, A Egner and S W Hell Proc. Nat. Acad. Sci. (USA) 97, 8206-8210 2000

[11] D A Armoogum, A J Bain and R J Marsh, Proc. SPIE-Int. Soc. Opt. Eng., 4812, 45-54 2002

[12] R Marsh, D A Armoogum and A J Bain, Chem. Phys. Lett. 366, 398-405 2002

[13] A J Bain, R J Marsh, D A Armoogum, L Porrès, O Mongin and M Blanchard-Desce, BioChem. Soc. Trans 31, 1047-1051 2003

[14] R. J Marsh, D A Armoogum and A J Bain, Proc. SPIE-Int. Soc. Opt. Eng., 5222, 32-44 2003

[15] M Albota D Beljonne, J -L Bredias, J E Erlich J -Y Fu, A A Heikal, S E Hess, T Kogej, M D Levin, S R Marder, D

McCord-Maughon, J W Perry, H Röckel, M. Rumi, G Subramaniam, W W Webb, X.-L Wu and C Xu, Science, 281, 1653-1656 1998

[16] M Barzoukas and M Blanchard-Desce, J. Chem. Phys. 113, 3951-3959 2000

[17] M Barzoukas and M Blanchard-Desce, Mol. Cryst. Liq. Cryst. Sci. Tech. 27, 2092001

[18] M Barzoukas and M Blanchard-Desce, Proc. SPIE 4461, 78-89 2001

[19] W H Lee, M Cho, S-J Leon and B R Cho, J. Phys. Chem. A 104, 11033-11040 2000

[20] L Ventelon, L Moreaux, J Mertz and M Blanchard-Desce, Chem. Commun. 2055-2056 1999

[21] L Ventelon, S Charier, L Moreaux, J Mertz and M Blanchard-Desce, Angew. Chem. Int. Ed. 40, 2098-2101 2001

[22] L Ventelon, L Moreaux, J Mertz and M Blanchard-Desce, Synth. Met. 127, 17-21 2002

[23] L Porrès, O Mongin, M Blanchard-Desce, L Ventelon, M Barzoukas, L Moreaux, T Pons, J Mertz Proc. SPIE-Int. Soc. Opt. Eng., 4797, 284-292 2002

[24] O Mongin L Porrès L Moreaux J Mertz and M Blanchard-Desce . Organic Letters 4, 719-722 (2002)

[25] O Mongin, L. Porrès, C. Katan, T. Pons, J. Mertz and M. Blanchard-Desce, Tetrahedron Letters 44, 8121-8125 (2003)

[26] S W Pauls, J F Hedstrom and C K Johnson, Chem. Phys., 237, 205-222 1998

[27] P R Callis J.Chem .Phys. 99, 27-37 1993

[28] A J Bain, P Chandna and G. Butcher, J Chem. Phys. 112, 10435-10449 2000

[29] R J Marsh, D A Armoogum, N Leonczek and A J Bain (to be published) 